DOI : 10.20287/doc.d24.en02

\title{
Irrintzina, le cri de la génération climat : un documentaire à la fois « singulier » et « pluriel »
}

\author{
Sandrine Ravel*
}

Selon Christel Taillibert, lorsqu'elles adoptent une rhétorique basée sur la fiction, les « images "engagées" peuvent - même si ce n'est pas le cas de l'ensemble d'entre elles - trouver leur public dans les salles d'exploitation commerciale. Ceci s'avère beaucoup plus rare et difficile lorsque la forme adoptée relève du genre documentaire. ${ }^{1}$

Or les scores d'entrées, récents et en salle, de Merci Patron (François Ruffin, 2016), La Sociale (Gilles Perret, 2016) ou encore Demain (Cyril Dion et Mélanie Laurent, 2015), ont montré que le long métrage militant, même sous une forme documentariste, s'extirpe des sentiers battus, plus confidentiels, Internet ou bien festivals, afin de saisir le grand public...

Force est de constater alors qu'Irrintzina, le cri de la génération climat enfonce le clou : car le film a été élu, sur AlloCiné, documentaire préféré des spectateurs en 2017 et une dizaine de mois après sa sortie, en novembre dernier, il est encore à l'affiche sur plus de vingt-cinq dates, en France... ${ }^{2}$ C'est pourquoi il vient d'attirer l'attention de France Culture, qui lui consacre une chronique dans Les Nouvelles de L'Éco...

D'ailleurs, certains documentaristes cités ci-dessus sont déjà des références pour son coauteur, Sandra Blondel, interviewé le 22 Mai 2018 à Bron, en France.

Sandra Bondel : À la fac, j'ai découvert et aimé le travail de Frederick Wiseman, Johan van der Keuken, Agnès Varda et Chris Marker. Aujourd'hui, je me sens particulièrement proche de cinéastes étiquetés "militants" comme Gilles Perret, Yannis Youlountas ou Jean-Paul Jaud, qui font un travail remarquable et nécessaire de lanceurs d'alertes et de mémoire des luttes sociales et écologistes...

* Université Lumière Lyon 2, PRCE titulaire du département Arts du Spectacle, Parcours Image, 69500 Bron, France. E-mail : Sandrine.Ravel@univ-lyon2.fr

1. TAILLIBERT Christel, «Les festivals aux prises avec l'engagement citoyen », Colloque international «L'engagement au cinéma », Université d'Angers / Université du Maine, Laboratoire 3L.AM, Angers, 2013.

2. Irrintzina, le cri de la génération climat, 2016. Blog : www.irrintzina-le-film.com/2016 /09/12/bonjour-tout-le-monde/ [consulté le 04 avril 2018]

Doc On-line, n. 24, setembro de 2018, www.doc.ubi.pt, pp. 318-324. 
Sandrine Ravel : La question du documentaire à la première personne va alors motiver cet entretien avec vous... Pour qui tout a commencé, cinématographiquement parlant, à la fois sur les bancs de l'université et en autodidacte, puisque vous comptez déjà à votre actif, depuis 2003, quelques opus engagés, coréalisés avec Pascal Hennequin, qui est aussi chef opérateur... Jusqu'à ce dernier, autoproduit (avec l'aide de plus de 50000 euros de financement participatif) et autodistribué...

SB : La production de ce film est à l'image du mouvement qu'il raconte, faite d'entraide et de solidarité !

SR : C'est d'ailleurs lors d'un séminaire annuel de la Fédération de l'audiovisuel participatif que Pascal Hennequin a rencontré, pour la première fois, Bizi!

Ce nom, qui signifie «Vivre! », en basque, est celui d'une organisation crée à l'initiative de quelques militants et qui a impulsé, sur tout l'hexagone et en quelques années, un ample mouvement citoyen, « des milliers de personnes », toujours selon Sandra, capables de s'opposer aux principaux responsables du dérèglement climatique....

Les deux coauteurs ayant, à leur tour, aidé au lancement d'un village Alternatiba, dans leur propre ville de Marseille...

Ces villages devaient constituer des alternatives écologiques : le pari a-t-il été tenu?

SB : Oui, ils ont tenu leur pari, c'est ce que nous racontons dans le film, qui est une immersion dans le monde du militantisme.

Bien que, dès le départ, nous leur avions bien spécifié que nous faisions notre documentaire de manière indépendante et que ce ne serait pas un outil piloté par les communicants du mouvement, nous leur avons montré les différentes étapes du montage à plusieurs reprises. Nous ne voulions pas trahir leur travail.

SR : L'aventure est entièrement filmée à l'épaule (pas de moyens supplémentaires pour un steadycam...) et, pour sa quasi-totalité, par Pascal Hennequin... À l'image, il y a d'ailleurs des prises de risque afin de réussir à suivre Bizi !... Par exemple, pour le Sommet du pétrole offshore de Pau : alors que les militants affrontent pacifiquement les forces de l'ordre, du gaz lacrymogène se répand sur l'objectif... Un C.R.S. dit même : «Recule, dégage ! » et la caméra ne recule pas...

SB : Bien sûr que j'ai vécu des moments de peur, mais les militants, de par leur propre courage, m'en ont donné et m'ont permis de dépasser mes limites. 
Car même si les portes nous avaient été ouvertes immédiatement, pendant le Tour Alternatiba notamment, à cause de conditions de tournage particulièrement difficiles, nous avons pensé arrêter le film à plusieurs reprises. Mais malgré des débuts un peu chaotiques, nous avons eu raison de persévérer!

SR : Lors du générique initial, c'est vous, Sandra, qui êtes créditée à la prise son et c'est aussi de cette manière que s'effectue votre première apparition dans le film... Quels étaient vos objectifs esthétiques sur le plan sonore?

SB : Avec des moyens très « low cost », c'était très difficile de tout faire en même temps : la prise de son, l'organisation du tournage, la régie etc. Très sincèrement, mon principal souci était d'avoir un son le plus "propre" possible au tournage. Pascal s'en est tout autant chargé, à certains moments, en plus de la caméra! Après oui, être le plus "vrai" possible, au plus prêt de la vie, était essentiel pour nous.

SR : Avant d'en venir au recours à la première personne du singulier, il faut savoir qu'il y a, dès l'ouverture du long métrage, la volonté de favoriser, dans un premier temps, celle du pluriel, le « nous »...

Ainsi, alors que prend place à l'image un fondu au noir, le spectateur entend, en off, un groupe de personnes qui scande : «Nous sommes tous des faucheurs de chaise. » De même à la fin du film; il y a une volonté de donner la parole aux militants, avant d'en faire une affaire personnelle...

Puis par la suite, il y aura une alternance voix off / discours des interlocuteurs, l'un des principes de base de ce documentaire : des spécialistes comme, par exemple, Txetx Etcheverry, co-fondateur de Bizi! et Altenatiba s'y expriment... Ou bien Christiane Hessel, marraine d'Alternatiba... Ou encore de nombreux responsables, comme par exemple Germain Sarhy, fondateur d'Emmaüs Lescar-Pau... Mais aussi, autre exemple, quatre bénévoles, parmi la quarantaine qui s'engage, de juin à septembre 2015, depuis Bayonne, dans un tour de France cycliste en 186 étapes... Des interlocuteurs comme autant de relais, issus d'univers très différents, mais qui font cause pour ces entretiens individualisés, lorsqu'ils ne sont pas restitués en off, la caméra filme à hauteur d'homme, se pose en plan fixe, au plus près de l'action, les mouvements se raréfiant... commune sur le plan écologique...

Puis le film bascule très rapidement, dès les premières séquences, pendant lesquelles débute le procès de Jon Palais à Dax, du « Nous », au « Je ».

C'est là que le spectateur vous voit, Sandra, depuis une fenêtre, capter le son de ce grand rassemblement militant, venu le soutenir... Vous illustrez ainsi et involontairement sans doute, les propos d'André Bazin, qui envisageait le cinéma comme une « fenêtre ouverte sur le monde »... 
Or, un cinéma-vérité, porté par des pères fondateurs aux Etats Unis, comme, entre autres exemples, Frederick Wiseman, auquel vous avez fait référence au début de cet article, ou bien Richard Leacock, existe, sans le recours à la voix off ; c'est pourquoi il est aussi connu sous le nom de « cinéma direct »...

SB : Côté esthétique j'aime beaucoup le travail du réalisateur autrichien Nikolaus Geyrhalter (Notre pain quotidien; Homo sapiens) qui réussit, par un travail formel singulier, à raconter des choses très puissantes de la folie autodestructrice de notre monde.

SR : « Singulier » est un mot, au moins, à double sens : en tant que synonyme d' « unique », «d'original » et en tant qu'antonyme de « pluriel »... Les deux conviennent à Irrintzina, mais c'est dans le cadre de cette deuxième acception que vous prenez la parole, à la première personne et en off, pour ne plus la lâcher...

SB : Au départ, il n'y avait pas de voix off prévue et puis, quand nous avons commencé à travailler avec Thomas Hakenholz sur le montage final, il nous est apparu évident qu'il fallait, pour ce film sur l'engagement, que je m'engage moi-même et que je parle de ma propre expérience. Comme nous avions encore la séquence du procès de Jon Palais à tourner, nous avons donc choisi de me filmer à ce moment-là et de monter le film sur un flash-back...

Il y a eu plusieurs étapes dans l'écriture et l'enregistrement de la voix off. C'était une première pour moi ! Jean-Marc Pisani, l'ingénieur du son et mixeur du film, avec qui nous l'avons enregistrée, m'a beaucoup aidée pour trouver « ma voix. » Nous avons fait un premier enregistrement vraiment pas bon du tout, pour lequel je n'ai pas réussi à trouver le ton (c'était beaucoup trop énergique...) Et puis, un deuxième : là, je me suis mise à raconter sur le ton de la « confidence »; j'étais alors beaucoup plus juste.

SR : Jusqu'à personnaliser cette cause, car vous confiez alors en off sur grand écran : «Au fil des mois, j'ai peu à peu fait corps avec cette lutte » : à la fois réalisatrice et personnage du film, vous revendiquez votre « Je », pour raconter et argumenter ouvertement...

SB : Je réalise des films, car c'est ma manière d'appréhender et de comprendre le monde. Chaque film marque une étape de mon cheminement et de ma vision du monde. J'assume totalement de faire du documentaire militant, tellement méprisé par la critique et dont l'objectif est de provoquer la mise en mouvement chez le spectateur. Mais j'espère ne pas rester enfermée dans ce genre! Aujourd'hui, j'aspire à entrer dans une autre phase de mon travail, chercher de nouvelles formes, toujours aussi politiques, mais peut-être moins 
militantes. Faire confiance aux situations et aux personnages, pour interroger, sans prendre position et chercher à convaincre le spectateur.

SR : Je sais que cela ne se fait pas de "demander son âge à une dame », mais si vous le souhaitez, vous pouvez me le donner... Quoi qu'il en soit, croyez-vous que la jeunesse de votre voix a également favorisé le succès du film auprès des lycéens, des étudiants ; le long-métrage étant, par exemple, le « coup de cœur » du journal Phosphore...?

SB : Je viens d'avoir 40 ans, donc je ne suis plus si jeune que ça! Mais oui, je pense que la jeunesse de ma voix aide les jeunes à rentrer dans l'histoire! Mais nous filmions aussi pour la «mémoire », pour constituer des archives et nous en avons fait don au mouvement; elles intéresseront peut-être un jour des chercheur-se-s.

SR : «Irrintzina » signifie « cri » en basque et donne le coup d'envoi du ralliement... Sachant que Bizi! veut sans doute toujours former, en un temps record, une nouvelle génération de militants, le film en a-t-il généré d'autres ?..

SB : Je pense qu'il y a une vitalité dans le film qui porte les spectateurs et donne envie de rejoindre le mouvement. C'est, en tout cas, les retours fréquents que nous avons à la suite des projections. Je vous confirme que c'est un outil de recrutement très efficace ! La voix off aide aussi le spectateur à s'identifier aux militants.

SR : Êtes-vous d'accord pour dire que le film a des allures de journal intime? De par l'utilisation de la première personne, mais aussi, dans un premier temps, de par le choix de circonstancier des faits relatifs à un périple de plus de trois ans, qui a commencé à Bayonne, en octobre 2013, au sein de Bizi ! Les séquences y sont contextualisées, des sous-titrages précisant date, année, jour et lieux...

SB : À mon sens, ce film est plus un récit initiatique qu'un journal intime, qui raconte cette prise de conscience là, cette urgence de s'engager et prendre parti dans un monde qui s'effondre. La caméra démarre de manière très observationnelle, puis, petit à petit, elle va se faire de plus en plus immersive pour devenir elle-même, à la fin, « acteur » du film («vous êtes filmé. »)

SR : Sur 1h40, il y a une vingtaine de reprises narratives, qui durent parfois plusieurs minutes, entrecoupées par des pauses... Deux mouvements les caractérisent alors : 
Le premier, qui va du début du film jusqu'à la COP 21 à Paris, s'en tient aux faits et aux personnes : ces narrations sont plutôt informatives, explicatives et se veulent objectives... Cependant, y a-t-il volonté de contrebalancer cette dimension omnisciente, au profit de narrations plus subjectives?

SB : Effectivement, on bascule progressivement d'une voix off plutôt descriptive à une voix off de plus en plus personnelle, jusqu'à la séquence des calanques après la COP21, où je parle du deuil et de mon sentiment d'être enfin en transition. J'ai d'ailleurs compris en faisant ce film la nécessité de cette fameuse «transition intérieure » que j' ai longtemps fustigée et pris conscience de l'aspect politique de la réappropriation de nos émotions.

SR : De fait, puisant dans le vif de votre histoire personnelle mouvementée au sein de Bizi !, le long métrage s'apparenterait alors à une sorte d'exutoire thérapeutique, un rapport particulièrement intime se créant alors entre narrateur et spectateur, autour d'une démarche autobiographique, qui réorganise le récit en un itinéraire physique et spirituel, un questionnement existentiel, voire métaphysique, vers un repérage, une découverte de soi («Je repense... »), au réconfort psychique : «C'est après la COP 21 que je suis sortie du déni... » N'est-ce pas là le véritable objectif de votre documentaire?

SB : Je crois que, pendant longtemps, nous avons souffert du syndrome des «bonnes nouvelles. » Nous ne voulions raconter que des récits «positifs » et « inspirants. » Nous n'avions pas du tout pris la mesure de ce que nous sommes en train de vivre et de la possibilité d'un effondrement systémique global. Nous n'avons pas compris l'urgence climatique, ni l'effondrement de la biodiversité, l'épuisement des ressources, l'appauvrissement des sols et tout le reste... La sortie du déni, pendant et depuis ce film, est très difficile à vivre. Nous sommes restés très light dans ce film par rapport à la question de l'effondrement.

SR : La caméra se fait alors plus hasardeuse : votre démarche, toujours artisanale, qui travaille avec un matériau vivant, se dirige vers des déambulations diurnes ou bien nocturnes, au grès des mises en place d'actions... La voix-off laisse entendre craintes, fragilités, le lexique abondant dans le sens du doute, sous le joug d'un manque de maîtrise, car rien n'est acquis d'avance... Par la même occasion, vous amoindrissez la puissance de votre «Je », d'auteure et de personnage qui est le votre, avec, à chaque fois, une instrumentalisation plus attristée de la musique extradiégétique...

SB : Ce film est comme une bouée de sauvetage, à laquelle je m'accroche aujourd'hui, pour ne pas sombrer. Les militants, que nous avons filmés pendant ces deux ans et avec qui j'ai partagé d'immenses moments de joie et de 
fraternité, me donnent l'espoir qu'ensemble nous pouvons donner le meilleur de nous-même, pour affronter les heures les plus sombres de notre histoire à venir.

SR : S'organiserait alors, sur l'ensemble du documentaire, un passage réussi d'un « Nous » introductif, puis alterné avec votre «Je », qui se fait de plus en plus présent, et de ce dernier vers, à nouveau, un « Nous », à la fois conclusif, ouvert et pluriel...

SB : Irrintzina raconte comment la transformation individuelle et collective s'alimente l'une l'autre. Comment nous sommes transformés par nos pratiques collectives et comment chacun peut transformer par un geste, une réflexion, des pratiques collectives. Cette question des va-et-vient entre le « je » et le «nous » est vraiment au centre de ce film. C'est ce que raconte aussi la citation qui ouvre le film : "C'est par l'action que notre imaginaire se transforme. »

SR : Dans ce long métrage, vous citez Pablo Servigne ainsi que Raphaël Stevens et leur traité intitulé : Comment tout peut s'effondrer-Petit manuel de collapsologie à l'usage des générations présentes (publié au Seuil, en 2015)... Pouvez-vous nous dire ce qu'est la collapsologie?

SB : L'objectif de la collapsologie est de décrire, analyser, comprendre l'effondrement de notre civilisation thermo-industrielle.

SR : J'avais aussi pensé à un « journal intime collapsologue » en voyant votre documentaire... Du moins, ce dernier se voudrait-il l'illustration, l'application de leur ouvrage?

SB : J'aime bien l'idée d'un "journal collapsologue", même si je sais que cet aspect de ma personne n'est pas du tout assumé dans ce film. D'une part, parce que c'était naissant pour moi à ce moment-là et puis, d'autre part, parce que le mouvement Alternatiba n'étant pas du tout raccord avec cette vision « catastrophiste », je n'ai donc pas voulu trop insister sur cet aspect, de peur de dénaturer leur action.

Par ailleurs, je ne crois pas que ce film soit l'illustration de leur ouvrage. Cela fera plutôt l'objet d'un prochain film !... 